\title{
Can the Choice of Radiotherapy Delivery Technique Influence Which Target Delineation Protocol to Use? A Plan-Quality-Based Analysis in Left Breast Cancer
}

\author{
${ }^{1}$ Department of Radiation Oncology, Rajiv Gandhi Cancer Institute \\ and Research Centre, Rohini, New Delhi, India \\ 2Department of Radiation Oncology, Sarvodaya Hospital and \\ Research Centre, Faridabad, Haryana, India \\ ${ }^{3}$ Department of Radiation Oncology, Illawarra Cancer Care Centre, \\ Wollongong, New South Wales, Australia \\ ${ }^{4}$ School of Medicine, Keele University, Staffordshire, \\ United Kingdom
}

Irfan Ahmad ${ }^{1, \odot}$ Kundan Singh Chufal ${ }^{1} \quad$ Chandi Prasad Bhatt ${ }^{2, \odot} \quad$ Alexis Andrew Miller ${ }^{3, \odot}$ Ram Bajpai,@ Rahul Lal Chowdhary ${ }^{1}$ Anjali Kakria Pahuja ${ }^{1}$ Akanksha Chhabra ${ }^{1}$ Munish Gairola ${ }^{1}$

\author{
Address for correspondence Kundan Singh Chufal, MD, \\ Department of Radiation Oncology, Rajiv Gandhi Cancer Institute \\ and Research Centre, Sector 5, Rohini, New Delhi 110085, India \\ (e-mail: kundan25@gmail.com).
}

\author{
Abstract \\ Keywords \\ - breast cancer \\ - European Society of \\ Radiation Therapy \\ - intensity modulated \\ radiotherapy \\ technique \\ - plan quality metric \\ - Radiation Therapy \\ Oncology Group \\ - radiotherapy \\ - volumetric modulated \\ arc therapy
}

Introduction This study investigates the optimal target delineation protocol stratified by treatment planning technique in patients undergoing whole breast radiotherapy after breast conservation surgery.

Materials and Methods Target delineation using Tangent (RTOG 0413 Whole Breast Irradiation Protocol), European SocieTy for Radiotherapy and Oncology (ESTRO), and Radiation Therapy \& Oncology Group (RTOG) guidelines was performed on 10 randomly selected treatment planning computed tomography datasets of patients with leftsided breast cancer. An objective plan quality metric (PQM) scoring schema was defined and communicated to the medical physicist prior to commencement of treatment planning. Treatment planning was performed using field-in-field (FiF) intensity modulated radiotherapy technique (IMRT), inverse IMRT, and volumetric modulated arc therapy (VMAT), for each type of target. Two-way repeated measures, analysis of variance was utilized to compare the total PQM scores and dosimetric variables, stratified by treatment planning method.

Results Total PQM score of plans for FiF, IMRT, and VMAT revealed that Tangent and ESTRO delineations were equivalent regardless of planning technique (Tangent vs. ESTRO for FiF, $p=0.099$; Tangent vs. ESTRO for IMRT, $p=0.029$; Tangent vs. ESTRO for VMAT, $p=0.438$ ). Both delineation protocols were significantly superior to RTOG for all treatment planning techniques.

Conclusion For all treatment planning techniques, ESTRO and Tangent delineation were equivalent and both achieved significantly higher scores than RTOG delineation. published online

December 30, 2020
DOI https://doi.org/

$10.1055 / \mathrm{s}-0040-1722428$

ISSN 2454-6798.
(C) 2020. Spring Hope Cancer Foundation \& Young Oncologist Group of Asia.

This is an open access article published by Thieme under the terms of the Creative Commons Attribution-NonDerivative-NonCommercial-License, permitting copying and reproduction so long as the original work is given appropriate credit. Contents may not be used for commercial purposes, or adapted, remixed, transformed or built upon. (https://creativecommons.org/licenses/by-nc-nd/4.0/).

Thieme Medical and Scientific Publishers Pvt. Ltd. A-12, 2nd Floor, Sector 2, Noida-201301 UP, India 


\section{Introduction}

Treatment planning for breast irradiation has evolved from conventional fields based on marks placed around the breast to modern three-dimensional computed tomography (3D CT)-based planning. Recognition of the late effects associated with breast irradiation is manifested primarily as an increased risk of long-term cardiac mortality, specifically in left-sided breast cancer. ${ }^{1,2}$ Technologically advanced radiotherapy delivery techniques have allowed a reduction in whole heart mean dose, which has emerged as the strongest predictor of radiation-induced cardiac mortality. ${ }^{3-5}$ Paralleling technological advances, guidelines have been formulated for the delineation of the whole breast target volume, though issues of interobserver variation persist. ${ }^{6-8}$ These parallel developments in target delineation and treatment delivery techniques have aided the evolution from conventional to 3D CT-based planning.

While the dosimetric performance of modern radiotherapy delivery techniques has been compared, meaningful interpretation is hampered by, but not limited to issues of type of target delineation performed, treatment planning proficiency and the large number of dosimetric parameters evaluated. As radiotherapy techniques and delineation guidelines continue to evolve, disentangling the confounding effects of both on each other may be difficult and is not currently addressed in the literature.

The purpose of this research was to determine the most optimal target delineation protocol for a given type of treatment planning technique, using an objective plan quality assessment tool.

\section{Materials and Methods}

We have previously reported the methodology for this analysis ( $\mathbf{- \text { Fig. }} \mathbf{1}$ ). ${ }^{9}$ In brief, we selected 10 left-sided breast cancer patients from our institutional database who had undergone breast conservation surgery and adjuvant radiotherapy (46Gy in 23Fx with an electron boost of $12.5 \mathrm{~Gy}$ in 5Fx to lumpectomy cavity). All patients had undergone a free-breathing CT scan on Siemens Somatom Sensation Open with a slice thickness of $2 \mathrm{~mm}$. Their DICOM CT datasets was retrieved, and target delineation was performed based on the RTOG 0413 (Tangent) Whole Breast Irradiation protocol, the RTOG (Radiation Therapy Oncology Group) consensus and ESTRO (European SocieTy of Radiation Therapy) consensus guidelines. ${ }^{6,710}$ Organs-at-risk (OAR), the left anterior descending (LAD) artery, and left ventricle (LV) were delineated as described in the RTOG 1005 protocol (NCT01349322) and a cardiac atlas, respectively. ${ }^{11}$

Subsequently, treatment planning was performed using field-in-field (FiF) intensity modulated radiotherapy technique (IMRT), inverse optimized IMRT, and volumetric modulated arc therapy (VMAT). ${ }^{12-19}$ To minimize interobserver variation, one radiation oncologist performed delineation of all structures on a single treatment planning system (Varian Eclipse v13.5, Varian Medical Systems) and a single medical physicist performed treatment planning on a single
TPS (Varian Eclipse v13.5; AAA algorithm, Varian Medical Systems, Palo Alto, CA, United States) and delivery platform (Varian TrueBeam v2.5; Millennium 120 MLC, Varian Medical Systems).

Plan quality assessment was performed using an objective plan quality metric (PQM) scale., ${ }^{9,20}$ Dosimetric data from each plan for each structure was extracted from exported DVH files using DVHmetrics v0.3.5 (www.rdocumentation. org/packages/DVHmetrics), per level 2 reporting recommendations by the ICRU Report $83 .^{21}$

Statistical comparison was performed using a two-way repeated measure, analysis of variance (ANOVA), after correcting for any possible interaction between target delineation protocol and planning technique in each ANOVA model. ${ }^{22}$ A Bonferroni correction was applied, and the significance level was set at $<0.005(0.05 / 9) .{ }^{23}$ All analyses were performed in Stata 14.2 SE (Statacorp Inc, College Station, TX, United States) statistical software.

\section{Results}

Ninety plans were selected from a pool of 450 generated plans on the basis of $\mathrm{PQM}$ score and minimum acceptance criteria. ${ }^{9}$ Descriptive data associated with PQM and dosimetric comparisons is provided in - Supplementary Material (available in the online version).

\section{PQM-Based Comparison of Delineation Guidelines}

For plans based on FiF IMRT, the combined PQM scores for Tangent delineation were equivalent to ESTRO delineation (Tangent vs. ESTRO, $p=0.099$ ) and both were significantly superior to RTOG delineation (Tangent vs. RTOG, $p<0.001$; ESTRO vs. RTOG, $p<0.001$ ) ( - Fig. 2). The subscore analysis revealed that the planning target volume (PTV) score for ESTRO delineation was higher than both RTOG and Tangent delineations (ESTRO vs. RTOG, $p<0.001$; ESTRO vs. Tangent, $p=0.001$ ), and that Tangent delineation was superior to RTOG delineation (Tangent vs. RTOG, $p=0.001$ ). The comparison of subscores for left lung, heart, and right breast revealed that ESTRO and Tangent delineations were equivalent and that both were superior to RTOG delineation.

For plans based on inverse optimized IMRT, the combined $\mathrm{PQM}$ scores for Tangent delineation were equivalent to ESTRO delineation (Tangent vs. ESTRO, $p=0.029$ ) and both were significantly superior to RTOG delineation (Tangent vs. RTOG, $p<0.001$; ESTRO vs. RTOG, $p<0.001$ ) (-Fig. 3). The subscore analysis for PTV score revealed that all delineation protocols were equivalent. The comparison of subscores for heart and right breast revealed that Tangent and ESTRO delineations were equivalent and both were superior to RTOG delineation. When comparing subscores for left lung, ESTRO delineation was superior to both RTOG and Tangent delineation protocols (ESTRO vs. RTOG, $p<0.001$; ESTRO vs. Tangent, $p=0.003$ ), and Tangent delineation was superior to RTOG delineation (Tangent vs. RTOG, $p=0.001$ ).

For plans based on VMAT planning technique ( - Fig. 4), the combined PQM scores for Tangent delineation were equivalent to ESTRO delineation (Tangent vs. ESTRO, $p=0.438$ ) and 


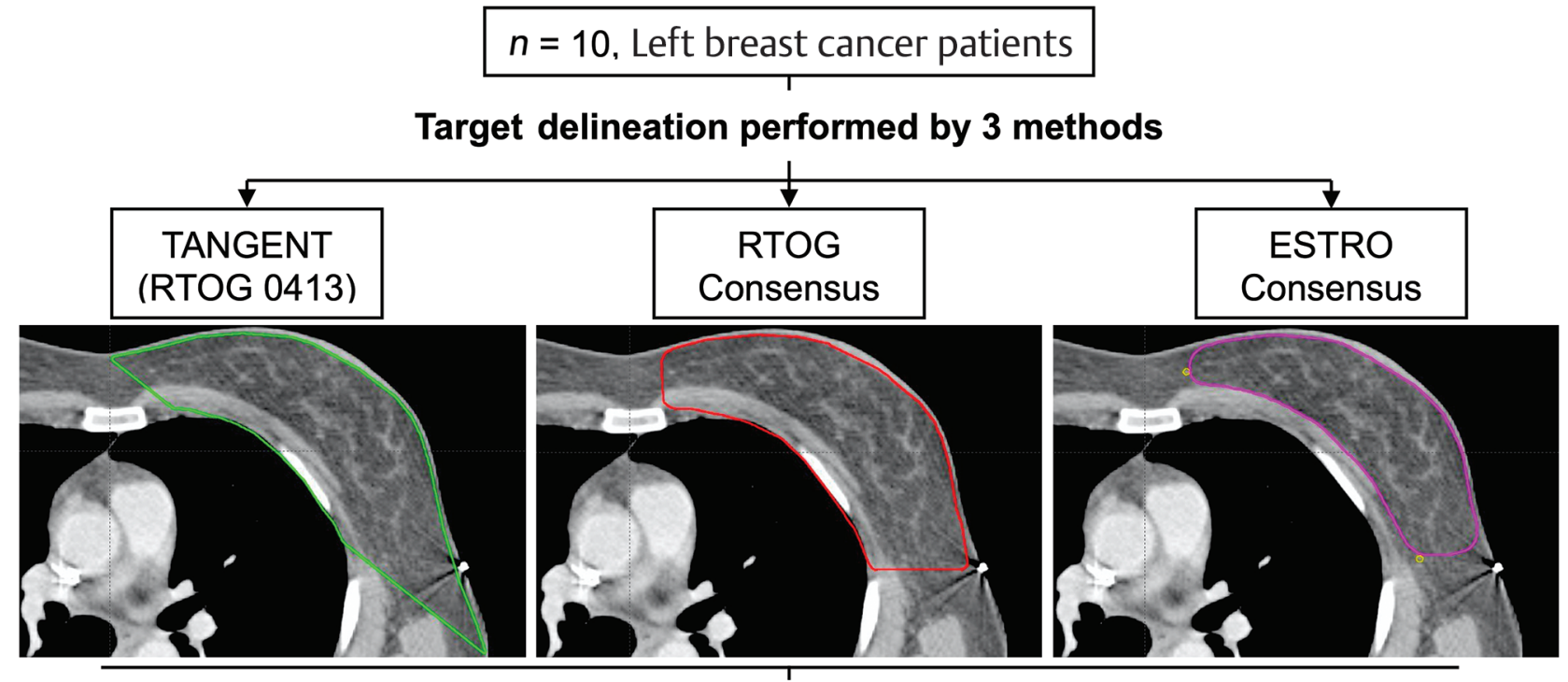

Each type of target planned by 3 techniques

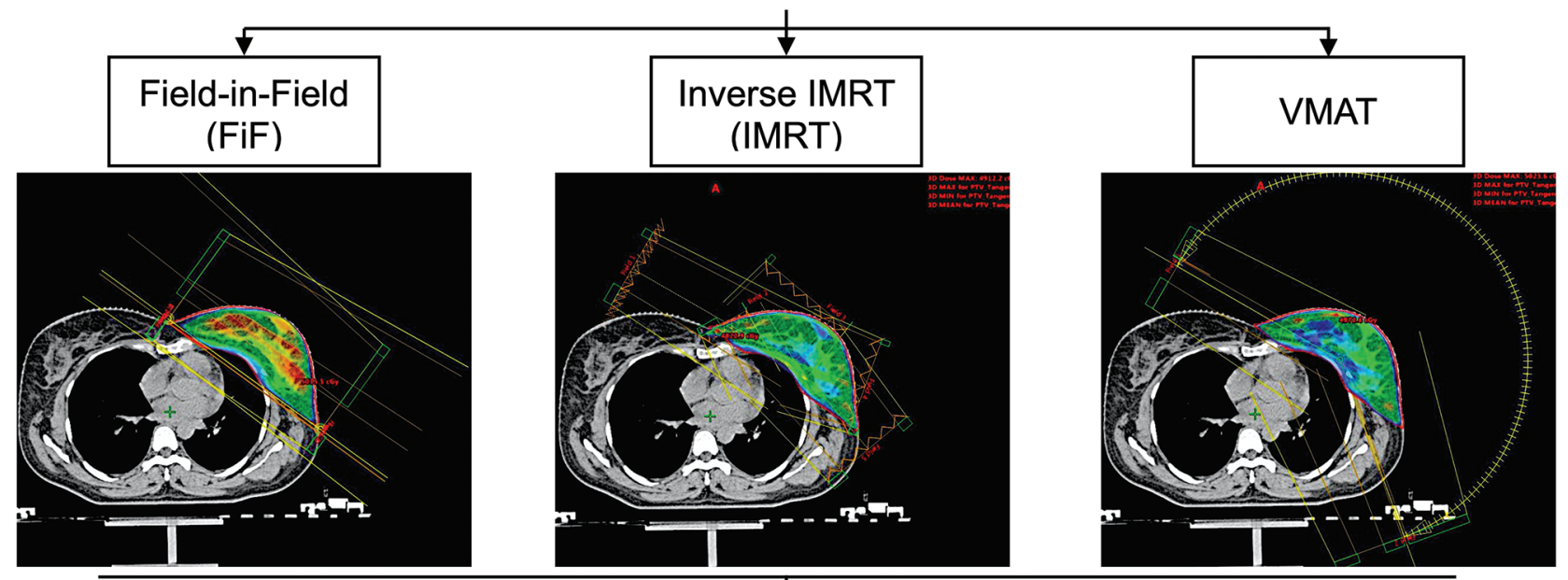

Maximum permissible iterations $=5$ per plan (Total planning time $<2$ days) out of which 1 was selected based on maximum PQM score Total plans generated for entire Cohort $=450$; Total plans analysed $=90$

\section{PQM Scoring}



Fig. 1 Study design. ${ }^{9}$ ESTRO, European SocieTy for Radiotherapy and Oncology; IMRT, intensity modulated radiotherapy technique; PQM, plan quality metric; RTOG, Radiation Therapy \& Oncology Group; VMAT, volumetric modulated arc therapy. 

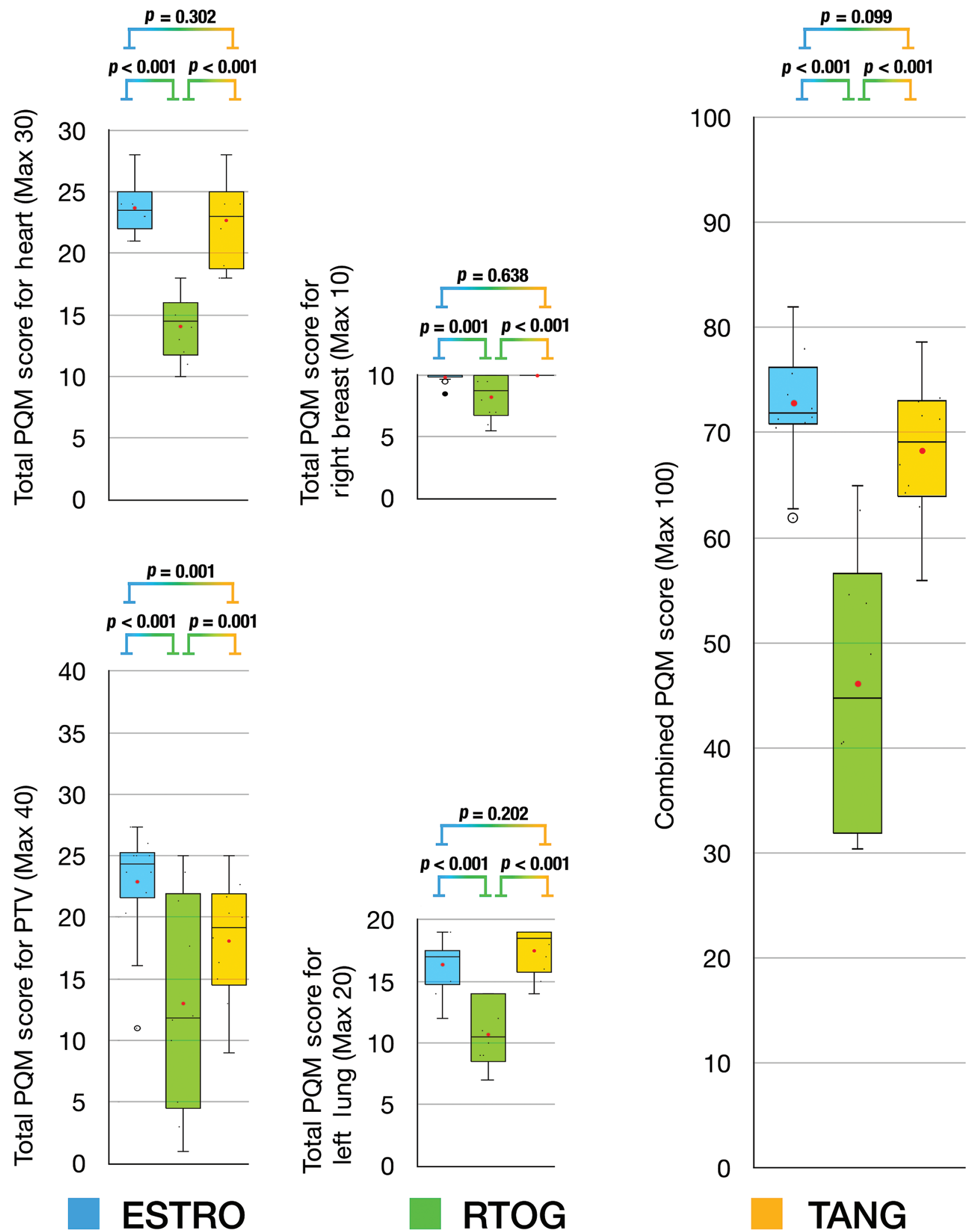

Fig. 2 Box and whisker plots for PQM score comparison of delineation protocols based on field-in-field planning technique. ESTRO, European SocieTy for Radiotherapy and Oncology; FiF, field-in-field IMRT; IMRT, intensity modulated radiotherapy technique; PQM, plan quality metric; RTOG, Radiation Therapy \& Oncology Group; TANG, RTOG 0413 (Tangent) Protocol; VMAT, volumetric modulated arc therapy.

both were significantly superior to RTOG delineation (Tangent vs. RTOG, $p=0.001$; ESTRO vs. RTOG, $p<0.001)(-$ Fig. 4). The subscore analysis for PTV score and right breast revealed that all delineation protocols were equivalent. When comparing 

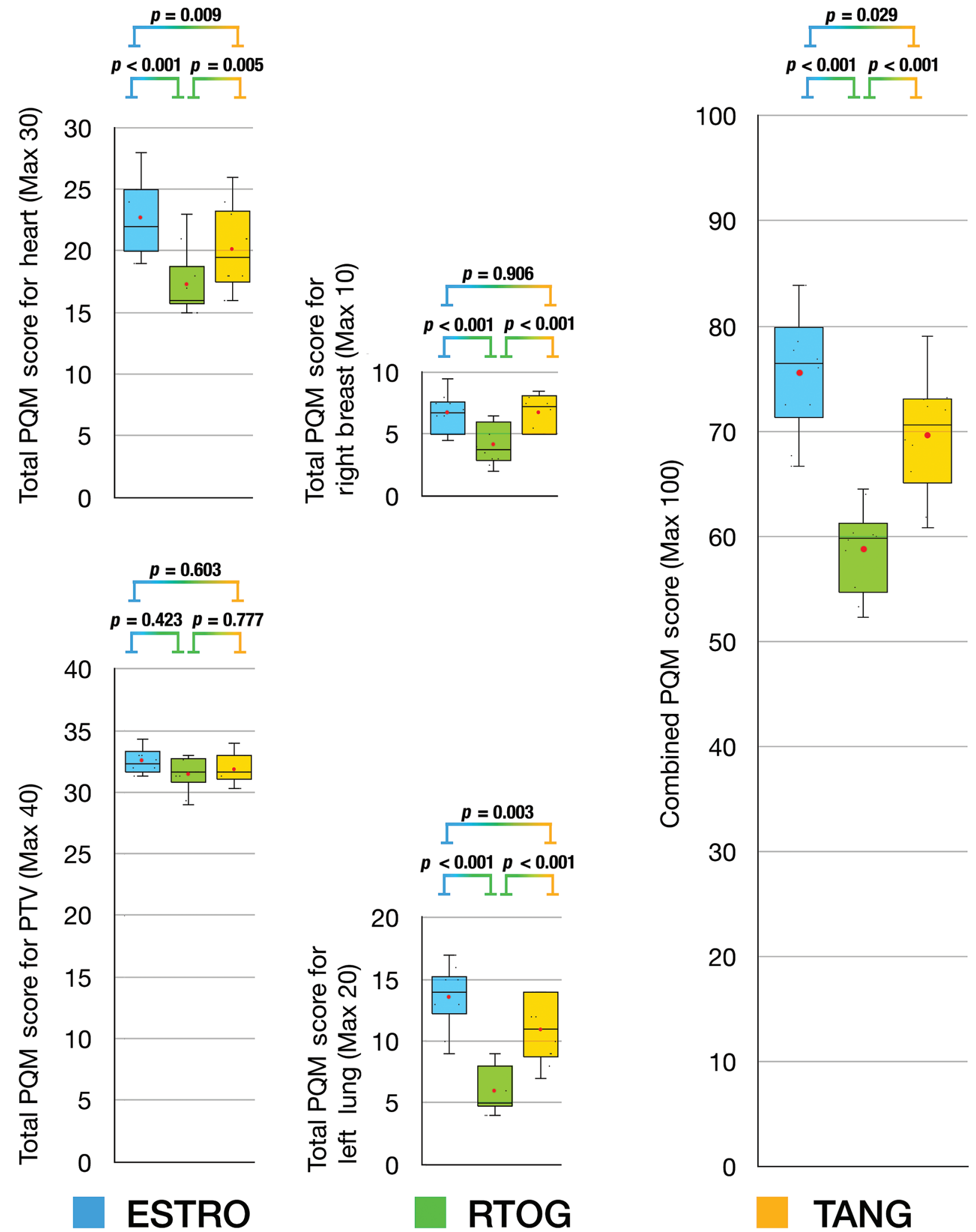

Fig. 3 Box and whisker plots for PQM score comparison of delineation protocols based on inverse optimized intensity modulated radiotherapy technique. ESTRO, European SocieTy for Radiotherapy and Oncology; FiF, field-in-field IMRT; IMRT, intensity modulated radiotherapy technique; PQM, plan quality metric; RTOG, Radiation Therapy \& Oncology Group; TANG, RTOG 0413 (Tangent) Protocol; VMAT, volumetric modulated arc therapy.

subscores for the heart, ESTRO and Tangent delineation protocols were equivalent (ESTRO vs. Tangent, $p=0.353$ ) and
RTOG was inferior to ESTRO (RTOG vs. ESTRO, $p=0.001$ ) but

equivalent to Tangent delineation protocol (RTOG vs. Tangent, 

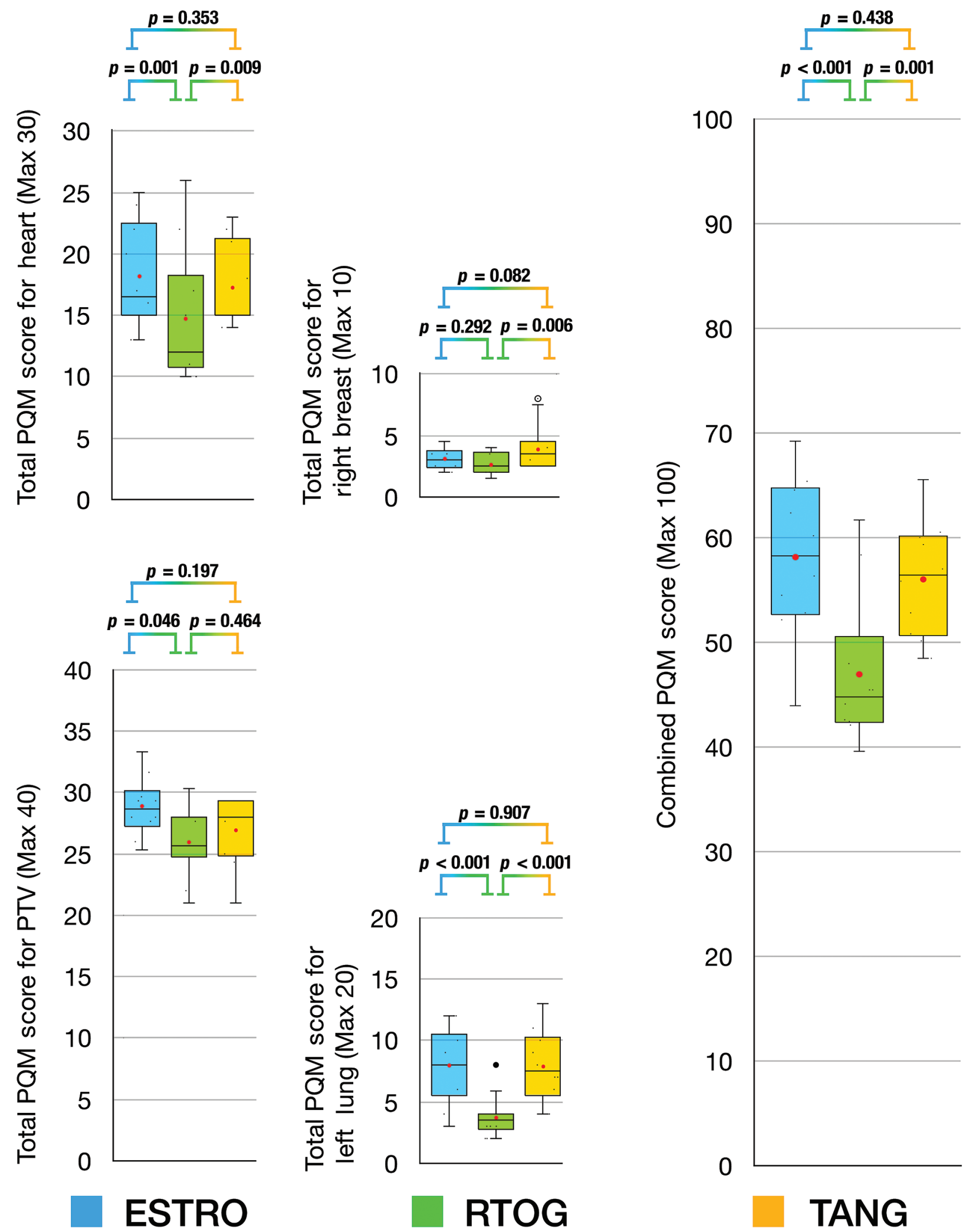

TANG

Fig. 4 Box and whisker plots for PQM score comparison of delineation protocols based on VMAT technique. ESTRO, European SocieTy for Radiotherapy and Oncology; FiF, field-in-field IMRT; IMRT, intensity modulated radiotherapy technique; PQM, plan quality metric; RTOG, Radiation Therapy \& Oncology Group; TANG, RTOG 0413 (Tangent) Protocol; VMAT, volumetric modulated arc therapy. 


\section{Dosimetric Comparison of Delineation Guidelines}

-Fig. 5 provides a summarized visual representation of the results of dosimetric comparison for all structures and dosimetric parameters analyzed (full descriptive data with associated $p$-values are provided in Supplemental Material, available in the online version). The data visualization technique chosen for comprehensively depicting results of all dosimetric comparisons is a modified version of the sunburst chart. ${ }^{24}$ Each analyzed structure and its associated dosimetric parameters form the origin of hierarchy, and three concentric circles represent the rank order, with the innermost circle being most inferior. Each dosimetric parameter has three radial arms representing planning technique, upon which the comparators (target delineation guidelines) are ranked. This method of qualitative data visualization was chosen because the values being compared were not on a uniform scale.

\section{PTV parameters (- Fig. 5A)}

Across all planning techniques, RTOG delineation protocol was either dosimetrically inferior to ESTRO delineation protocol (12/24 comparisons) or the overall comparison between all delineation protocols was not statistically significant (11/24 comparisons). The comparisons for tangent delineation protocol were more heterogeneous. Tangent delineation was in nearly equal measures dosimetrically superior to (7/24 comparisons) or equivalent to RTOG delineation (6/24 comparisons).

\section{Heart, LAD artery, and LV parameters (- Fig. 5B-D)}

For the whole heart contour, across almost all dosimetric parameters and planning techniques, RTOG delineation was dosimetrically inferior to both ESTRO and Tangent delineation (23/30 comparisons), except $\mathrm{D}_{2 \%}$ for IMRT planning where it was dosimetrically equivalent to Tangent delineation. Except for the comparisons where there was no statistically significant difference amongst all delineations (6/30 comparisons), ESTRO delineation was dosimetrically equivalent to Tangent delineation across all planning techniques and dosimetric parameters (23/30 comparisons).

The dosimetric comparisons for LAD revealed that RTOG delineation was dosimetrically inferior to ESTRO delineation (12/21 comparisons) and Tangent delineation was dosimetrically equivalent to ESTRO delineation (9/21 comparisons). Overall in 9/21 comparisons, there was no statistically significant difference among all delineations.

Similarly, almost all dosimetric comparisons for the LV demonstrated that RTOG delineation was dosimetrically inferior to ESTRO delineation (16/21 comparisons), irrespective of planning technique. Tangent delineation was dosimetrically equivalent to ESTRO delineation for most comparisons (15/21 comparisons), except $\mathrm{D}_{2 \%}$ with IMRT technique where it was dosimetrically inferior.

\section{Left lung ( - Fig. 5E)}

For the left lung, across all planning techniques and almost all dosimetric parameters, RTOG delineation protocol was dosimetrically inferior to ESTRO delineation protocol (23/24 comparisons). Tangent delineation protocol was dosimetrically equivalent to ESTRO delineation protocol for almost all comparisons (20/24 comparisons) and consequently dosimetrically superior to RTOG delineation for almost all comparisons (22/24 comparisons).

\section{Right breast ( - Fig. 5F)}

Except for two comparisons $\left(\mathrm{V}_{2}\right.$ and $\mathrm{V}_{3}$ for VMAT planning) where ESTRO delineation was dosimetrically equivalent to RTOG delineation, across all planning techniques and almost all comparisons for the right breast, either RTOG delineation was dosimetrically inferior to both ESTRO and Tangent delineations (16/24 comparisons), or there was no significant difference between delineation protocols (6/24 comparisons).

\section{Discussion}

We believe that this study is the first to analyze the performance of contouring protocols when viewed from the perspective of the medical physicist (which is the most optimal target delineation technique for a particular planning technique?). Our results suggest that irrespective of the planning technique employed, total PQM scores for ESTRO and Tangent delineation were equivalent and both achieved significantly higher scores than RTOG delineation.

Our results on the performance of delineation guidelines can be explained by the shared similarities of Tangent and ESTRO delineation guidelines. The principle behind the ESTRO guideline is that target delineation should not result in volumes larger than "conventional" simulator-based RT volumes. ${ }^{7}$ A notable difference of the ESTRO guideline is to restrict the dorsal border at the pectoralis major muscle and gradually move ventrally near the caudal edge to exclude subcutaneous abdominal fat. However, despite this modification in the ESTRO guidelines, the heart subscores between Tangent and ESTRO delineations were not significantly different (despite the Tangent delineation including the chest wall). The known issue of variation in the medial-lateral direction influencing OAR dose can explain the performance of the RTOG delineation, though delineation was in strict adherence to the published guideline. ${ }^{8}$ Furthermore, our study did not permit any contour modification after the plans were obtained, in contrast to common clinical practice. ${ }^{8}$

Our analysis can be criticized as being antithetical to modern radiotherapy practice with limited applicability, as planning technique is determined by target delineation and not vice-versa. We want to emphasize that our objective was purely exploratory and the intent of which was to empirically compare delineation protocols, controlling for as many variables as possible. Obviously, the gold standard for comparing delineation protocols would be clinical data on local failures. Data on regional nodal failures stratified by delineation protocols is now emerging, and while our analysis was not designed to assess the quality of regional nodal irradiation, this is an important avenue for research and our group will be addressing it in the near future. ${ }^{25}$

Our analysis shows that if a particular planning technique is preferred, some thought should be given to the delineation protocol best suited for it. At this time, in the absence of a local control analysis, it is impossible to decide whether ESTRO or RTOG delineation guidelines should be followed, and until such a report appears, radiation oncologists can 

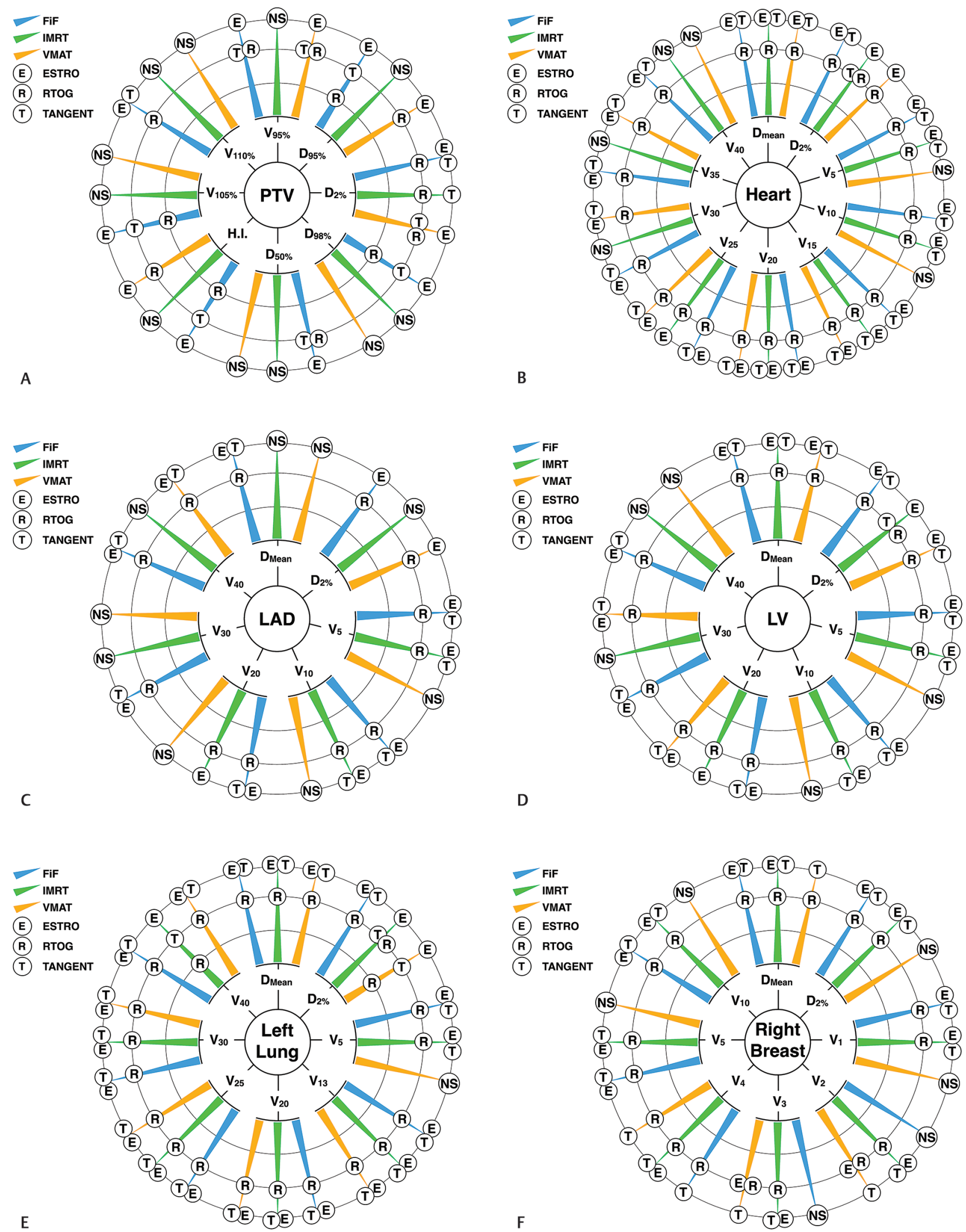

Fig. 5 Modified sunburst charts of dosimetric comparisons, depicting the ordinal performance of delineation guidelines stratified by planning technique (please refer - Supplementary Material, available in the online version, for descriptive data). (A) Planning target volume (PTV), (B) heart, (C) left anterior descending (LAD) artery, (D) left ventricle (LV), (E) left lung, and (F) right breast. 
choose either. The results of this analysis and a companion analysis $^{9}$ support our conclusion that either FiF or IMRT when coupled with Tangent or ESTRO delineation protocol yields the most optimal combination of target coverage and OAR sparing. We therefore advise against the routine use of RTOG delineation or VMAT planning technique purely on plan quality and dosimetric grounds and await the results of clinical trials utilizing the RTOG delineation guidelines. Just as planning techniques are sympathetic to delineation protocols, delineation protocols can also be sympathetic to planning techniques.

\section{Conflict of Interest}

None declared.

\section{References}

1 Darby S, McGale P, Correa C, et al; Early Breast Cancer Trialists' Collaborative Group (EBCTCG). Effect of radiotherapy after breast-conserving surgery on 10-year recurrence and 15-year breast cancer death: meta-analysis of individual patient data for 10,801 women in 17 randomised trials. Lancet 2011;378(9804):1707-1716

2 Darby S, McGale P, Peto R, Granath F, Hall P, Ekbom A. Mortality from cardiovascular disease more than 10 years after radiotherapy for breast cancer: nationwide cohort study of 90 000 Swedish women. BMJ 2003;326(7383) :256-257

3 Yeboa DN, Evans SB. Contemporary breast radiotherapy and cardiac toxicity. Semin Radiat Oncol 2016;26(1):71-78

4 Darby SC, Ewertz M, McGale P, et al. Risk of ischemic heart disease in women after radiotherapy for breast cancer. $N$ Engl J Med 2013;368(11):987-998

5 Taylor CW, Wang Z, Macaulay E, Jagsi R, Duane F, Darby SC. Exposure of the heart in breast cancer radiation therapy: a systematic review of heart doses published during 2003 to 2013. Int J Radiat Oncol Biol Phys 2015;93(4):845-853

6 White J, Tai A, Arthur D, et al. Radiation Therapy Oncology Group (RTOG). Breast cancer atlas for radiation therapy planning: consensus definitions. Available at: https://www.rtog. org/CoreLab/ContouringAtlases.aspx. Accessed 31st July 2020.

7 Offersen BV, Boersma LJ, Kirkove C, et al. ESTRO consensus guideline on target volume delineation for elective radiation therapy of early stage breast cancer. Radiother Oncol 2015;114(1):3-10

8 Li XA, Tai A, Arthur DW, et al; Radiation Therapy Oncology Group Multi-Institutional and Multiobserver Study. Variability of target and normal structure delineation for breast cancer radiotherapy: an RTOG Multi-Institutional and Multiobserver Study. Int J Radiat Oncol Biol Phys 2009;73(3):944-951

9 Ahmad I, Chufal KS, Bhatt CP, et al. Plan quality assessment of modern radiotherapy delivery techniques in left-sided breast cancer: an analysis stratified by target delineation guidelines. BJR Open 2020;2:20200007

10 Jain AK, Vallow LA, Gale AA, Buskirk SJ. Does three-dimensional external beam partial breast irradiation spare lung tissue compared with standard whole breast irradiation? Int J Radiat Oncol Biol Phys 2009;75(1):82-88
11 Feng M, Moran JM, Koelling T, et al. Development and validation of a heart atlas to study cardiac exposure to radiation following treatment for breast cancer. Int J Radiat Oncol Biol Phys 2011;79(1):10-18

12 Donovan EM, Bleackley NJ, Evans PM, Reise SF, Yarnold JR. Dose-position and dose-volume histogram analysis of standard wedged and intensity modulated treatments in breast radiotherapy. Br J Radiol 2002;75(900):967-973

13 Mukesh MB, Barnett GC, Wilkinson JS, et al. Randomized controlled trial of intensity-modulated radiotherapy for early breast cancer: 5-year results confirm superior overall cosmesis. J Clin Oncol 2013;31(36):4488-4495

14 Tanaka H, Hayashi S, Hoshi H. Determination of the optimal method for the field-in-field technique in breast tangential radiotherapy. J Radiat Res (Tokyo) 2014;55(4):769-773

15 Sasaoka M, Futami T. Dosimetric evaluation of whole breast radiotherapy using field-in-field technique in early-stage breast cancer. Int J Clin Oncol 2011;16(3):250-256

16 Freedman GM, Li T, Nicolaou N, Chen Y, Ma CC, Anderson PR. Breast intensity-modulated radiation therapy reduces time spent with acute dermatitis for women of all breast sizes during radiation. Int J Radiat Oncol Biol Phys 2009;74(3):689-694

17 Badakhshi H, Kaul D, Nadobny J, Wille B, Sehouli J, Budach V. Image-guided volumetric modulated arc therapy for breast cancer: a feasibility study and plan comparison with three-dimensional conformal and intensity-modulated radiotherapy. Br J Radiol 2013;86(1032) :2013051510.1259/ bjr.20130515

18 Fogliata A, Seppälä J, Reggiori G, et al. Dosimetric trade-offs in breast treatment with VMAT technique. $\mathrm{Br}$ J Radiol 2017;90(1070):20160701 10.1259/bjr.20160701

19 Cozzi L, Lohr F, Fogliata A, et al. Critical appraisal of the role of volumetric modulated arc therapy in the radiation therapy management of breast cancer. Radiat Oncol 2017; 12(1):20010.1186/s13014-017-0935-4

20 Nelms BE, Robinson G, Markham J, et al. Variation in external beam treatment plan quality: an inter-institutional study of planners and planning systems. Pract Radiat Oncol 2012;2(4):296-305

21 The International Commission on Radiation Units and Measurements. Prescribing, recording, and reporting photon-beam intensity-modulated radiation therapy (IMRT): report 83. J ICRU 2010;10:1-106

22 Kuehl RO, Design of Experiments: Statistical Principles of Research Design and Analysis. London: Duxbury-Thomson Learning; 2000

23 Shaffer JP. Multiple hypothesis testing. Annu Rev Psychol 1995;46:561-584

24 Stasko J, Zhang E, Focus+Context Display and Navigation Techniques for Enhancing Radial, Space-Filling Hierarchy Visualizations. INFOVIS 2000;57-65. DOI:10.1109/INFVIS. 2000.885091

25 Loganadane G, Truong PT, Taghian AG, et al. Comparison of nodal target volume definition in breast cancer radiation therapy according to RTOG Versus ESTRO Atlases: a practical review from the TransAtlantic Radiation Oncology Network (TRONE). Int J Radiat Oncol Biol Phys 2020;107(3):437-448 